\title{
Experimental Characterization of Orientation Formation in a Mold Cavity with Sudden Contraction
}

\author{
Kristian A. Olivero And M. CEngIZ Altan* \\ School of Aerospace and Mechanical Engineering \\ University of Oklahoma \\ Norman, OK 73019
}

\begin{abstract}
Orientation formation of short fibers suspended in a flow through a planar mold cavity is experimentally analyzed. The suspension of corn syrup mixed with short fibers is injected into the mold at constant flow rate through a narrow, planar inlet gate. The mold cavity contains a sudden expansion following the inlet gate, and a three-to-one sudden contraction downstream. Orientation data are obtained using computerized image analysis on photographs of thirteen zones of interest near the sudden contraction. The photographs are taken through the transparent top mold wall, thus collecting average planar orientation data through the mold thickness. Orientation distribution histograms are generated from the individual fiber orientations to graphically depict orientation microstructure. These histograms reveal bimodal fiber orientations in nearly all zones of interest. Experiments performed at different volume flow rates and viscosities also confirmed the bimodal orientation distribution observed through the top mold wall. Orientation results are observed to be more random compared to those predicted using Jeffery's theory (He et al., 1997). Fiber size or wall effects may contribute to the formation of a more random orientation field.
\end{abstract}

\section{INTRODUCTION}

NJECTION MOLDING IS among the most commonly used methods to manufacture short-fiber-reinforced composite materials. During injection molding processes, the flow field in the mold cavity causes the fibers to translate and rotate resulting in a complex orientation field in the molded part. The mechanical properties of the molded part are highly dependent on orientation field; thus, models to characterize this flow induced orientation field are needed. Accurate experimental characteriza-

*Author to whom correspondence should be addressed. 
tion of orientation field is also necessary to assess the predictive capability of these models.

Several experimental studies on the orientation formation of short fibers in a viscous flow have been conducted. Initial experimental studies observed the motion of individual fibers traveling through an experimental flow configuration. For example, Murty and Modlen (1977) and Lee and George (1978) investigated the flow kinematics, rotation of fibers, and formation of stagnant eddies in convergent channels. Vincent and Agassant (1985) studied fiber rotation in a diverging channel flow. Experimental observation of individual fibers is useful for comparison with theory based on the dynamics of a single fiber, but may not be adequate to construct a detailed description of orientation field formed by a large number of fibers.

Bay and Tucker (1992a) performed an experimental study of orientation field in center-gated disk and film-gated strip mold cavities. Their study involved the injection molding of nylon $6 / 6$ reinforced with $43 \mathrm{wt} \%$ glass fibers. Sections were cut from the molded parts and polished. Cross sections of fibers on the polished faces were digitized and analyzed to obtain orientation data for each fiber. From this data, the second-order moment of the orientation probability density function (i.e., second-order orientation tensor), $\mathrm{S}_{\mathrm{ij}}$, was calculated as described by Bay and Tucker (1992b) at each location. The second-order orientation tensor is commonly used to represent both experimental and theoretical microstructure, and is shown to completely characterize orientation field in dilute, non-Brownian suspensions of spheroidal particles (Rao, 1996). Although the process used to obtain the data provided by Bay and Tucker (1992a) is rather involved, the results presented are comprehensive and useful for comparison with theoretical models.

In addition to experimental studies, numerical prediction of orientation field in injection molded parts is studied by a number of researchers. Theoretical studies to predict orientation field are based mostly on the rotation of a hydrodynamically isolated ellipsoidal particle immersed in a viscous Newtonian flow (Jeffery, 1922). Initially, Altan et al. (1990) presented numerical prediction of three-dimensional fiber orientation in Hele-Shaw flows, thus simulating orientation formation in injection mold filling. Some of the other studies predicting orientation structure in injection molding have been published by Matsuoka et al. (1990), Bay and Tucker (1992c), De Frahan et al. (1992), Freidrichs et al. (1993), Chung and Kwon (1995), Altan and Rao (1995), and Chung and Kwon (1996).

In this study, orientation formation in a steady Newtonian flow through a planar mold cavity containing a three-to-one sudden contraction is experimentally studied. Computerized image analysis is performed on photographs of thirteen zones of interest near the sudden contraction. Orientation results for nine zones are presented in the form of orientation distribution histograms. Histograms for different filling conditions are also presented to depict the effect of Reynolds number on the orientation field. 


\section{EXPERIMENTAL SETUP AND DATA ANALYSIS}

Filling experiments are performed using the experimental setup shown in Figure 1. The suspension is injected into a planar mold cavity using a Masterflex I/P 73 peristaltic pump. The microprocessor-controlled pump can be set to provide constant flow rates ranging from 0.25 to $8.11 \mathrm{~L} / \mathrm{min}$ for water. Experiments are performed at pump settings of $0.25,0.50$, and $1.00 \mathrm{~L} / \mathrm{min}$ for water, which yield $10-50 \%$ lower flow rates due to the higher viscosity of the corn syrup suspension used in this study. The flow oscillations caused by the peristaltic pump are minimized by an in-line pulse dampener. Tygon tubing having an inside diameter of 9.5 $\mathrm{mm}(3 / 8 \mathrm{inch})$ is used throughout the setup. The suspension is stored in a feed reservoir and pumped through the experimental mold cavity. After flowing through the cavity, the suspension is returned to the reservoir to facilitate continuous operation at steady conditions.

For each experiment, the corn syrup is diluted with water to a selected viscosity ranging from 300 to $2000 \mathrm{cp}$. Viscosity measurements are made with a Brookfield DVII+ viscometer. A specific gravity cup with $0.5 \%$ accuracy is used to obtain the density of the corn syrup and fibers. The densities of the undiluted corn syrup and fibers are found to be $1400 \mathrm{~kg} / \mathrm{m}^{3}$ and $770 \mathrm{~kg} / \mathrm{m}^{3}$, respectively. Short fibers are prepared by hand cutting longer fibers with $0.17 \mathrm{~mm}$ diameter to an average length of

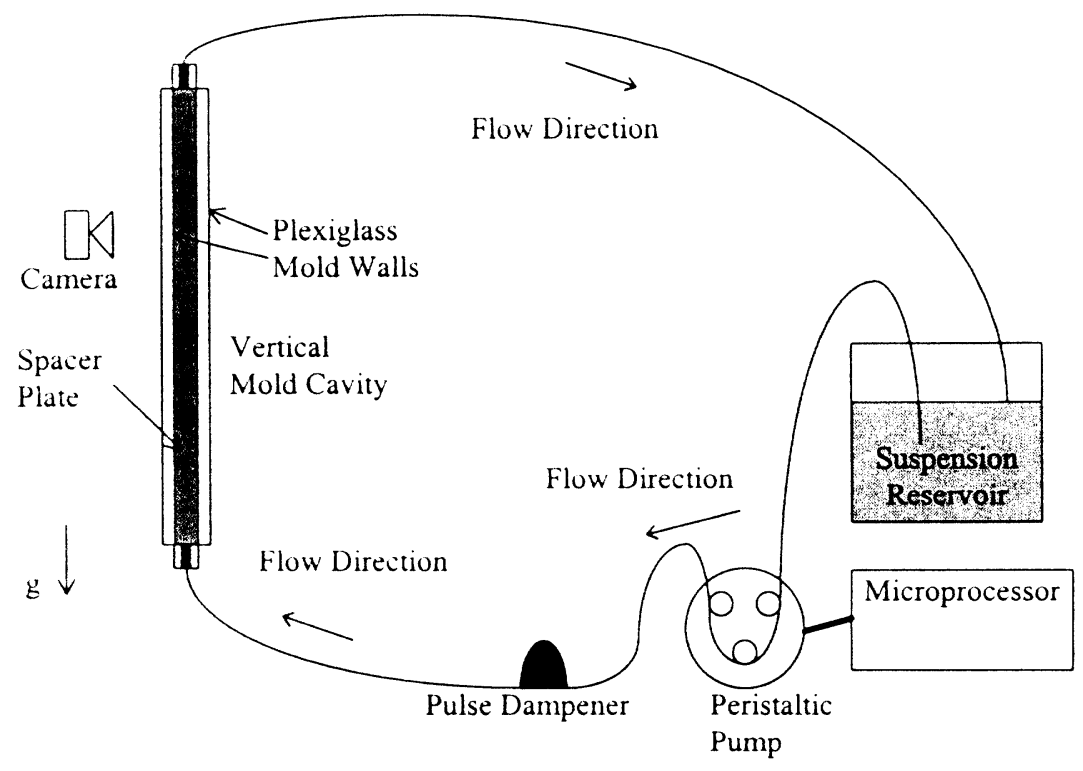

Figure 1. Experimental molding setup used to collect fiber orientation data. 
$1.55 \mathrm{~mm}$. This leads to an average aspect ratio of 9.14 . Suspensions are prepared having less than $0.2 \%$ fibers by weight $(0.36 \%$ by volume). The fibers are slowly mixed with the corn syrup solution to ensure uniform dispersion.

Three different Reynolds numbers for the experimental conditions can be defined. At the fiber length scale, two microscale Reynolds numbers are defined in terms of the fiber diameter, $D$, and average fiber length, $L_{\text {ave }}$

$$
\operatorname{Re}_{D}=\frac{\rho V_{\max } D}{\mu} \text { and } \operatorname{Re}_{L}=\frac{\rho V_{\max } L_{\text {ave }}}{\mu}
$$

where $V_{\max }$ is the maximum midplane velocity at a particular zone of interest. The minimum and maximum microscale Reynolds numbers for the experimental conditions are $\mathrm{Re}_{L \max }=0.004$ and $\mathrm{Re}_{\text {Dmin }}=0.0002$ downstream of the sudden contraction. These values are much less than unity, justifying comparisons with the numerical orientation predictions based on Jeffery's theory. The third Reynolds number is defined at macroscale in terms of the flow rate $Q$ and half gap width $h$.

$$
\operatorname{Re}=\frac{\rho Q}{\mu h}
$$

The experimental conditions lead to a macroscopic Reynolds number in the range of 0.12 to 0.28 . These values are less than unity, thus indicating the presence of Hele-Shaw flow.

The planar mold cavity is formed by placing an aluminum spacer plate between two sheets of plexiglass. The spacer plate, and thus the cavity, has a thickness of $6.35 \mathrm{~mm}(1 / 4 \mathrm{inch})$. The suspension is injected into the cavity through an inlet gate $25.4 \mathrm{~mm}$ ( $1 \mathrm{inch})$ in width, specially designed to achieve a planar velocity profile. After the inlet gate, the suspension undergoes a sudden expansion to a mold 228.6 $\mathrm{mm}$ (9 inch) in width. At $279.4 \mathrm{~mm}$ (11 inches) downstream, a three-to-one sudden contraction down to $76.2 \mathrm{~mm}$ ( 3 inches) takes place. The suspension exits the mold through a gate identical to the inlet gate. The fibers are buoyant and rise to the top wall in a horizontally positioned mold. Thus, the entire mold is aligned vertically to minimize gravity effects on the orientation field. The mold geometry, the dimensions, and the locations of the thirteen zones of interest are shown in Figure 2.

Data are collected after the mold is completely filled and steady conditions are established. Photographs of fibers in thirteen $25.4 \mathrm{~mm}$ ( 1 inch) square zones of interest near the sudden contraction are taken through transparent mold walls. The photos are digitized, and computerized image analysis is performed on each picture to obtain the planar orientation of each fiber. Figure 3 is an example of a digitized photograph of fibers in zone 12. In Hele-Shaw flows, fibers have predominantly 


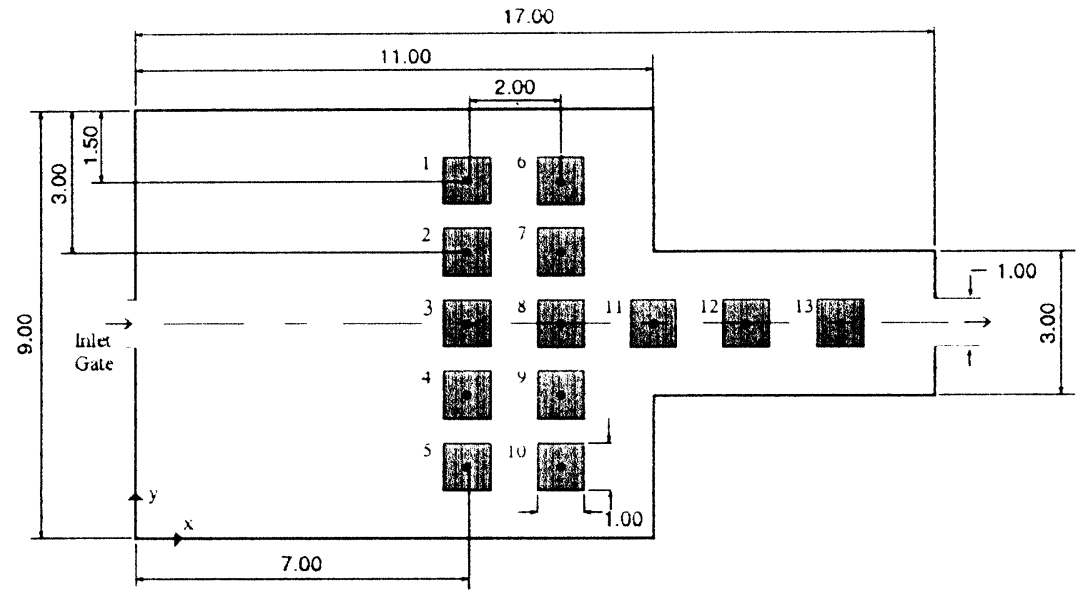

Figure 2. Mold geometry, dimensions (in inches), and thirteen zones of interest.

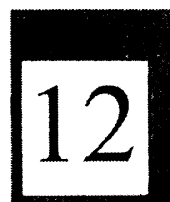

量

Figure 3. Photograph of zone 12, a 1-inch square zone of interest following the sudden contraction. 
in-plane orientations; thus, the planar orientation data may be adequate to describe the orientation structure. The photographs contain fibers through the mold thickness, leading to orientation results averaged through the mold cavity. Several photographs of each location are superimposed to reduce statistical variations by increasing the total number of fibers in each zone. Typically three photographs of each zone are used, yielding a total of 200-300 fibers.

The second-order orientation tensor, $\mathrm{S}_{\mathrm{ij}}$, is currently used most often to characterize the orientation field. In this study, $S_{i j}$ is found to be inadequate to represent the experimental data. Reasons for this include averaging microstructure through the mold thickness and bimodal orientation distributions. Rather than utilizing a higher order orientation tensor, fiber orientation data is presented in the form of radial orientation distribution histograms. The circumferencial axis represents orientation angles $\left(0^{\circ}\right.$ indicating the direction of the centerline of the mold), and the radial axis represents the number of fibers in each orientation interval. The orientation angles are divided into $30^{\circ}$ intervals. Smaller intervals give jagged curves, and larger intervals do not give enough resolution to examine orientation details. The two ends of each fiber are indistinguishable; thus, the histograms have angular symmetry with a period of $180^{\circ}$. Similar radial orientation histograms using $20^{\circ}$ angular intervals are given by Olivero et al. (1997) for a lower volume flow rate. Olivero et al. compared numerically predicted and experimentally measured eigenvalues of the second-order orientation tensor at these zones of interest. The comparisons, despite depicting similar trends, showed considerable quantitative differences.

\section{RESULTS}

Figures 4-12 are radial orientation distribution histograms for nine zones of interest near the sudden contraction (results for zones 4, 5, 9, and 10 are omitted due to mold symmetry). The location of each zone is shown in Figure 2. Zones 1-10 are upstream of the sudden contraction, and zones 11-13 are downstream of the contraction. Results in Figures 4-12 are obtained using a suspending fluid having a viscosity $\mu=1200 \mathrm{cp}$ injected at a flow rate of $\mathrm{Q}=7.5 \times 10^{-6} \mathrm{~m}^{3} / \mathrm{s}$. In each zone, orientation results are compared with orientation predictions given by $\mathrm{He}$ et al. (1997). He et al. numerically solved the Hele-Shaw flow for the same mold geometry. Using this flow, the orientation field was calculated based on Jeffery's theory, and the average orientation structure through the thickness was given at thirteen zones of interest. It is also illustrative to see the direction perpendicular to the local flow on orientation histograms. Thus, the preferred fiber orientation based on Jeffery's theory and the direction perpendicular to the local flow at that zone are superimposed on each orientation histogram.

Orientation data for zone 1 is shown in Figure 4. A bimodal distribution is clearly observed with peaks near the predicted preferred orientation and in the direction perpendicular to the local flow. Figure 5 indicates a higher degree of fiber align- 


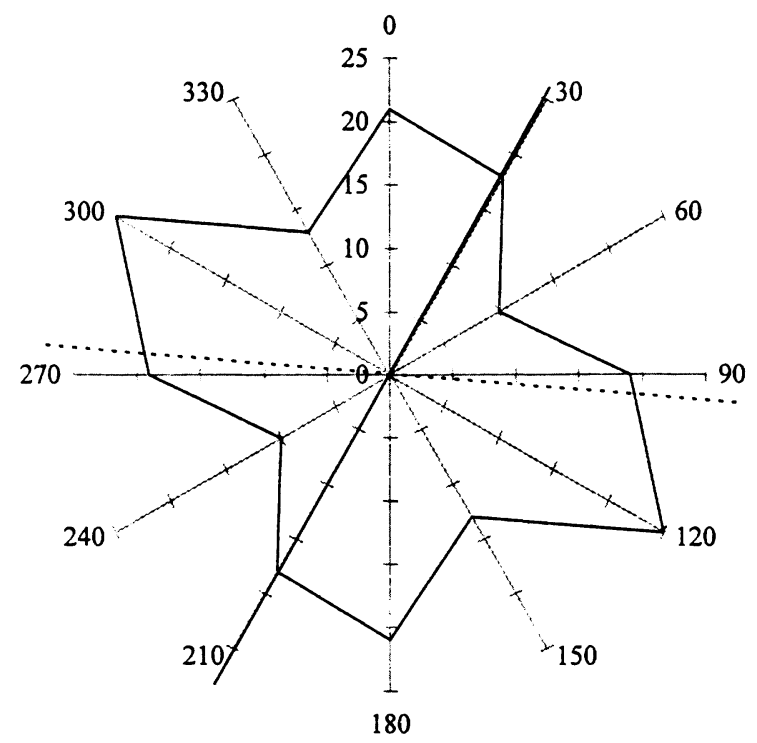

Figure 4. Planar orientation distribution histogram for zone $1, Q=7.5 \times 10^{-6} \mathrm{~m}^{3} / \mathrm{s}$, $\mu=1200 \mathrm{cp}$. - Jeffery's angle; . . Perpendicular to local flow direction.

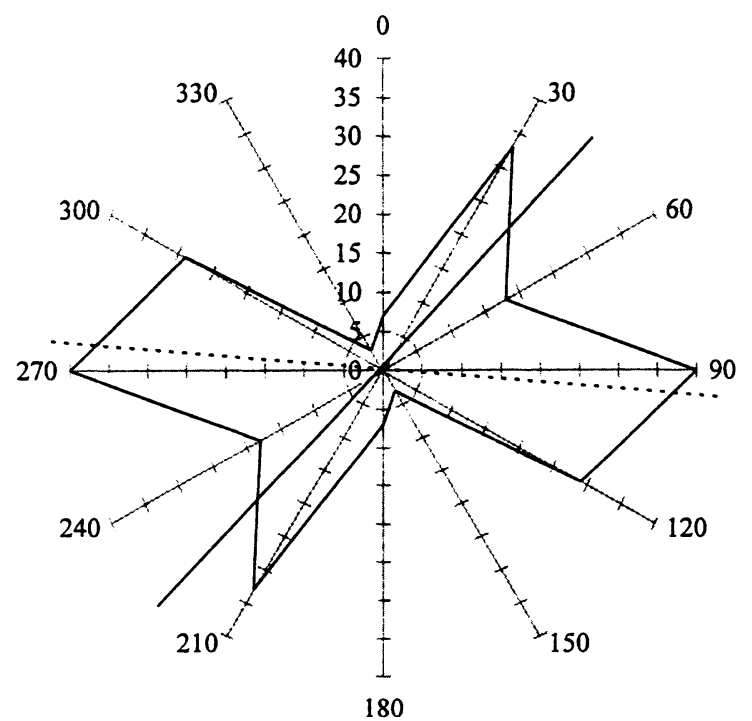

Figure 5. Planar orientation distribution histogram for zone $2, Q=7.5 \times 10^{-6} \mathrm{~m}^{3} / \mathrm{s}$, $\mu=1200 \mathrm{cp}$. - Jeffery's angle; - - Perpendicular to local flow direction. 


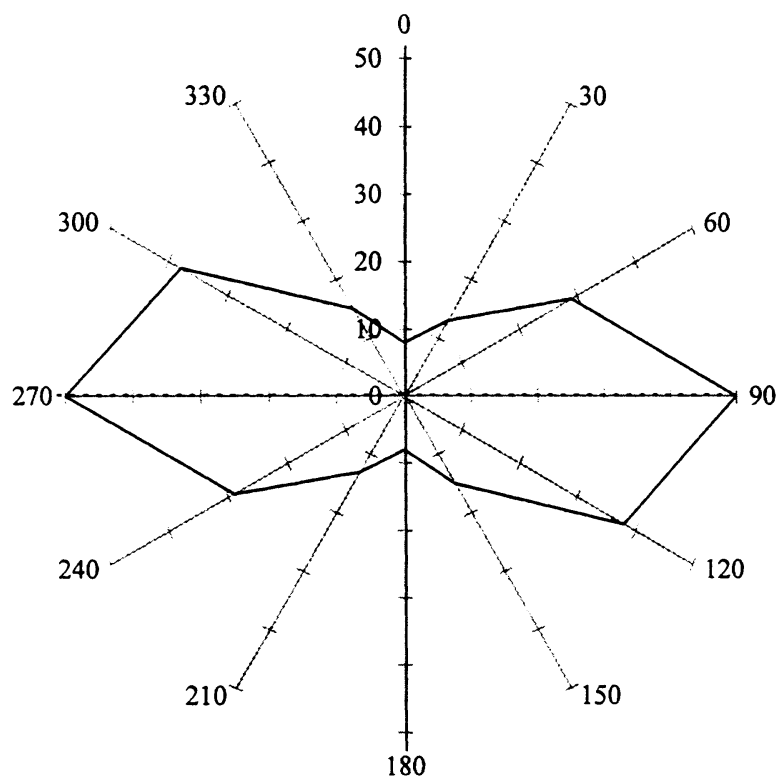

Figure 6. Planar orientation distribution histogram for zone $3, Q=7.5 \times 10^{-6} \mathrm{~m}^{3} / \mathrm{s}, \mu=1200 \mathrm{cp}$. - Jeffery's angle; - . - Perpendicular to local flow direction.

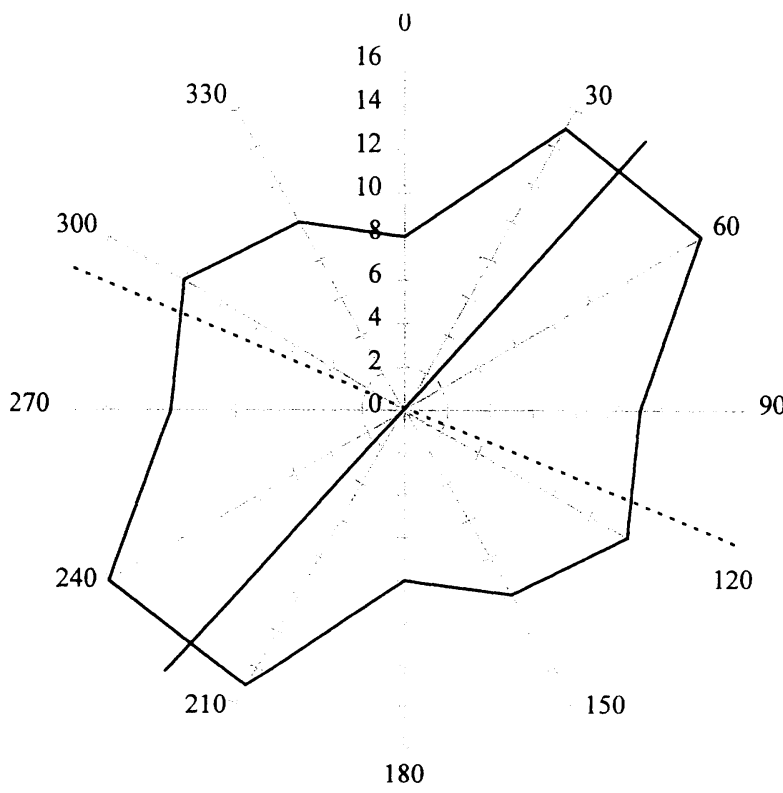

Figure 7. Planar orientation distribution histogram for zone $6, Q=7.5 \times 10^{-6} \mathrm{~m}^{3} / \mathrm{s}, \mu=1200 \mathrm{cp}$. - Jeffery's angle; - - - Perpendicular to local flow direction. 


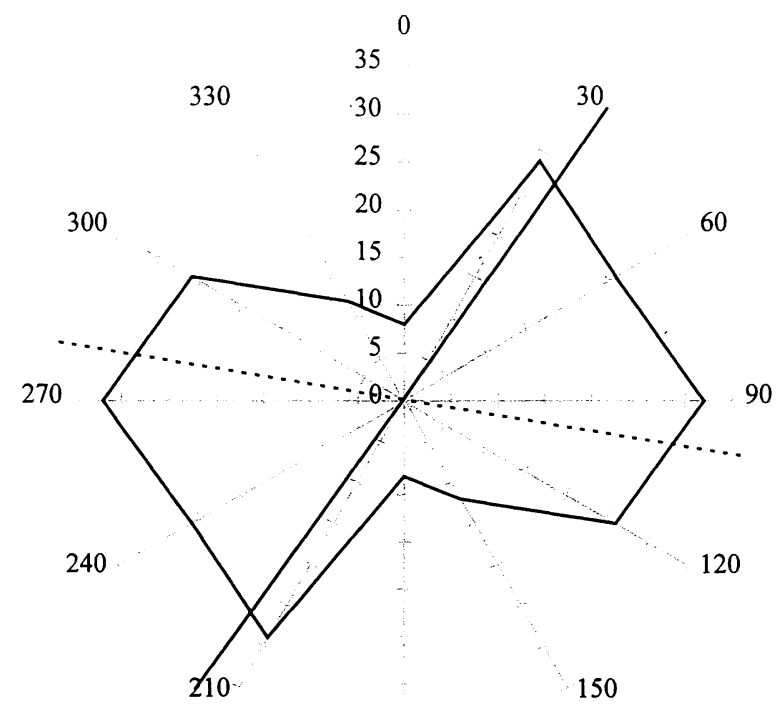

180

Figure 8. Planar orientation distribution histogram for zone $7, Q=7.5 \times 10^{-6} \mathrm{~m}^{3} / \mathrm{s}, \mu=1200 \mathrm{cp}$. - Jeffery's angle; - . - Perpendicular to local flow direction.

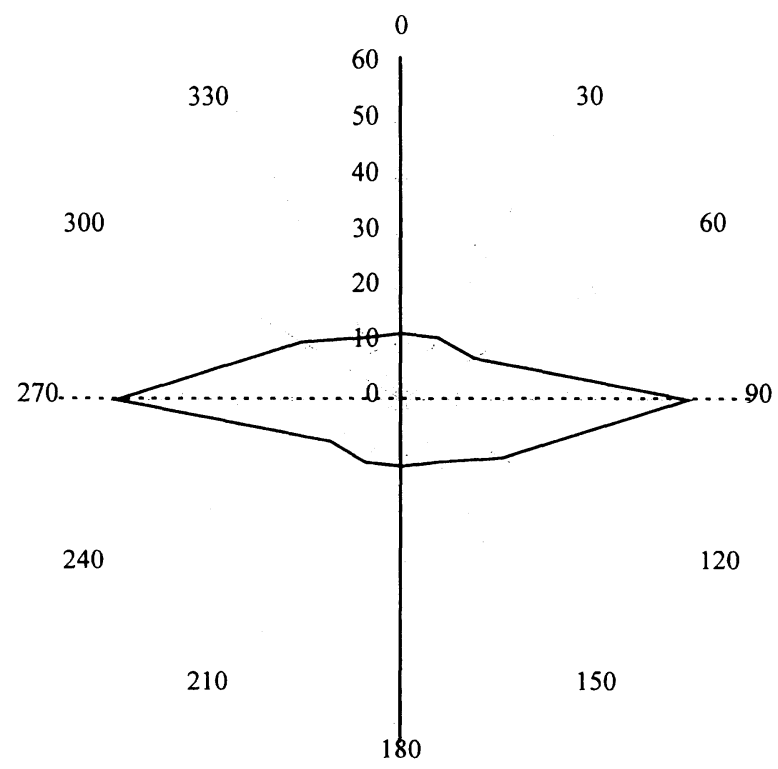

Figure 9. Planar orientation distribution histogram for zone $8, Q=7.5 \times 10^{-6} \mathrm{~m}^{3} / \mathrm{s}, \mu=1200 \mathrm{cp}$. - Jeffery's angle; - - - Perpendicular to local flow direction. 


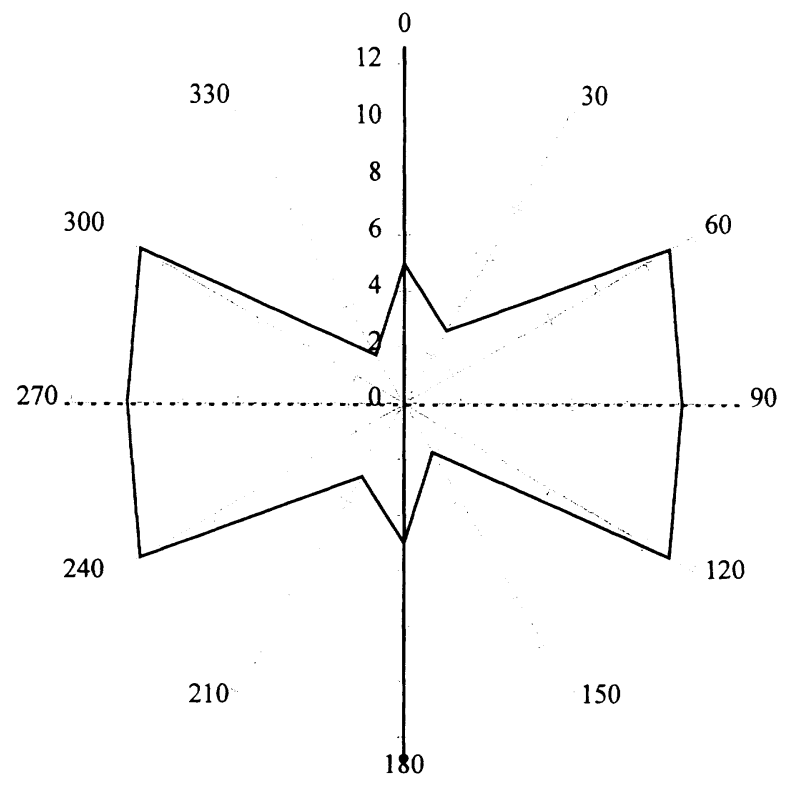

Figure 10. Planar orientation distribution histogram for zone $11, Q=7.5 \times 10^{-6} \mathrm{~m}^{3} / \mathrm{s}$, $\mu=1200 \mathrm{cp}$. - Jeffery's angle; - - Perpendicular to local flow direction.

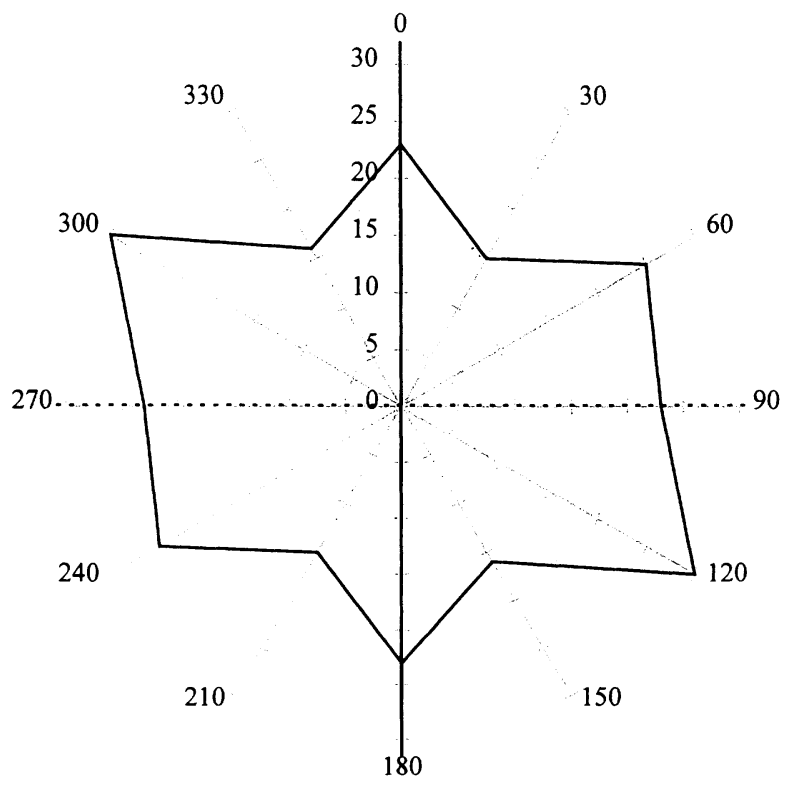

Figure 11. Planar orientation distribution histogram for zone $12, Q=7.5 \times 10^{-6} \mathrm{~m}^{3} / \mathrm{s}$, $\mu=1200 \mathrm{cp}$. - Jeffery's angle; . - Perpendicular to local flow direction. 


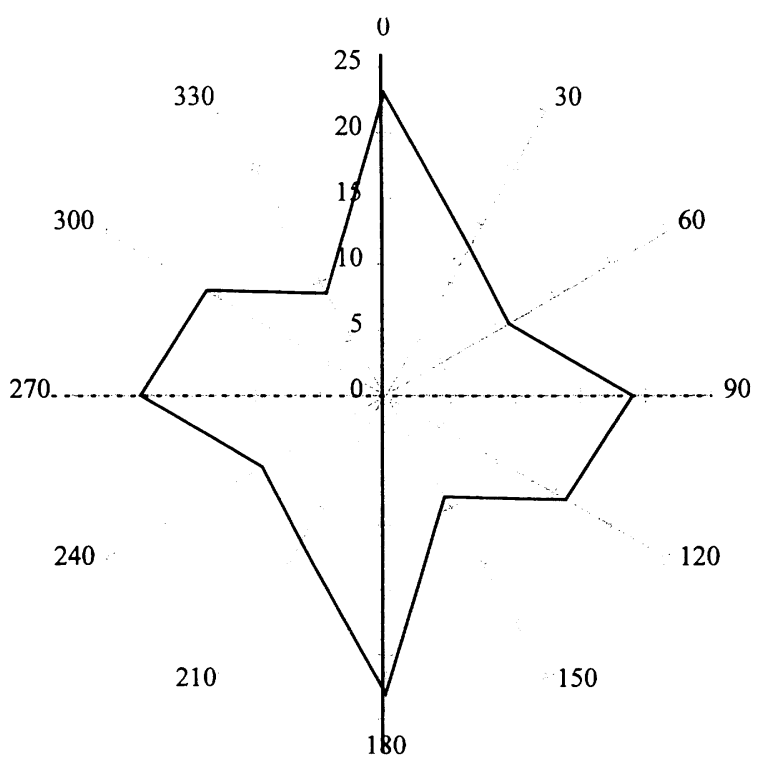

Figure 12. Planar orientation distribution histogram for zone $13, Q=7.5 \times 10^{-6} \mathrm{~m}^{3} / \mathrm{s}$, $\mu=1200 \mathrm{cp}$. - Jeffery's angle; - - Perpendicular to local flow direction.

ment in zone 2. Bimodal alignment peaks are more pronounced compared to zone 1. The first orientation peak is near the calculated preferred orientation. The second peak is near perpendicular to the flow direction. Orientation data from zone 3 is shown in Figure 6. This zone is on the symmetry axis, and fibers are observed to align predominantly perpendicular to the flow direction. Orientation results for zone 6, shown in Figure 7, depict fiber alignment primarily in the predicted preferred orientation. The orientation distribution, however, has a smaller second alignment peak near perpendicular to the flow direction. Zone 7 is closer to the symmetry axis than zone 6 . Figure 8 shows more distinct bimodal distribution in this zone compared to zone 6 . Alignment peaks are observed in the predicted preferred orientation and near perpendicular to the flow. Figure 9 shows the orientation histogram for zone 8 , which is on the symmetry axis just prior to the sudden contraction. Although Jeffery's theory predicts alignment in the flow direction in zone 8 , fibers are seen to align predominantly perpendicular to the local flow. Figure 10 shows the orientation distribution for zone 11 located at the sudden contraction. Fibers are mostly aligned perpendicular to the flow direction, but this alignment is not as substantial as that of zone 8. A small alignment peak in the flow direction (i.e., theoretical preferred orientation) is apparent. The orientation histogram for zone 12 is shown in Figure 11. The histogram depicts a somewhat random orientation distribution with a slight alignment peak along the predicted orientation (i.e., in the flow direction). Fibers are more aligned in the flow direction than those 
in zone 11, which is consistent with the theory. Zone 13, shown in Figure 12, is the final zone before the exit gate. As predicted by the theory, the fibers are primarily aligned in the flow direction; however, a smaller second orientation peak exists perpendicular to the local flow direction.

The experimental data for zones 1-3 and zones 6-8 indicate an increasing degree of alignment in the direction predicted by Jeffery's theory with increasing distance from the symmetry axis. In zones 3 and 8 (on the mold's symmetry axis), the fibers are aligned predominantly perpendicular to the flow, with a low degree of alignment in the predicted preferred orientation direction. In zones 2 and 7 (slightly away from the mold's symmetry axis) fibers are mostly aligned perpendicular to the local flow, with distinct alignment peaks in the predicted orientation directions. In zones 1 and 6 the degree of alignment in the predicted direction is even stronger.

The theory predicts increasing fiber alignment in the flow direction as the fibers move through the sudden contraction along the symmetry axis. Zones 3 and 8 show fiber alignment predominantly perpendicular to the flow direction. In zone 11, however, an alignment peak in the flow direction becomes evident. As fibers move through zones 12 and 13, the alignment in the flow direction increases as predicted by Jeffery's theory.

Bimodal orientation distributions are observed in all the experiments performed at various low Reynolds numbers, which are obtained by changing the volume flow rate and suspension viscosity. For example, Figures 8, 13, and 14 depict orientation distributions in zone 7 for different Reynolds numbers. Data from these three experiments show similar bimodal peaks. The first peak is observed along the predicted preferred orientation, whereas the second peak is perpendicular to the local flow.

It should be noted that the Jeffery's angle superimposed on the orientation histograms is taken from the numerical results of He et al. (1997). He et al. calculated the orientation field throughout the mold cavity at five equally spaced layers through the cavity thickness (i.e., at 0, 20, 40,60, and $80 \%$ of the distance from the cavity midplane to the wall). Results from these layers are extrapolated to the top wall and then averaged at each zone of interest to obtain a representative orientation structure through the thickness. The through-thethickness average orientation is described by a second-order tensor from which the Jeffery's angle is determined. The numerical method, as described in detail by Rao (1996), accounts for planar as well as through-the-thickness velocity gradients and solves for the three-dimensional second-order orientation tensor without closure approximations. In addition, Rao (1996) showed that for dilute suspension flows, the second-order tensor completely describes the flowinduced orientation structure at a particular point. Thus, the principal directions have been obtained by the accurate implementation of Jeffery's theory without additional approximations or simplifying assumptions about the flow kinematics. Considering the experimental conditions described earlier (i.e., 


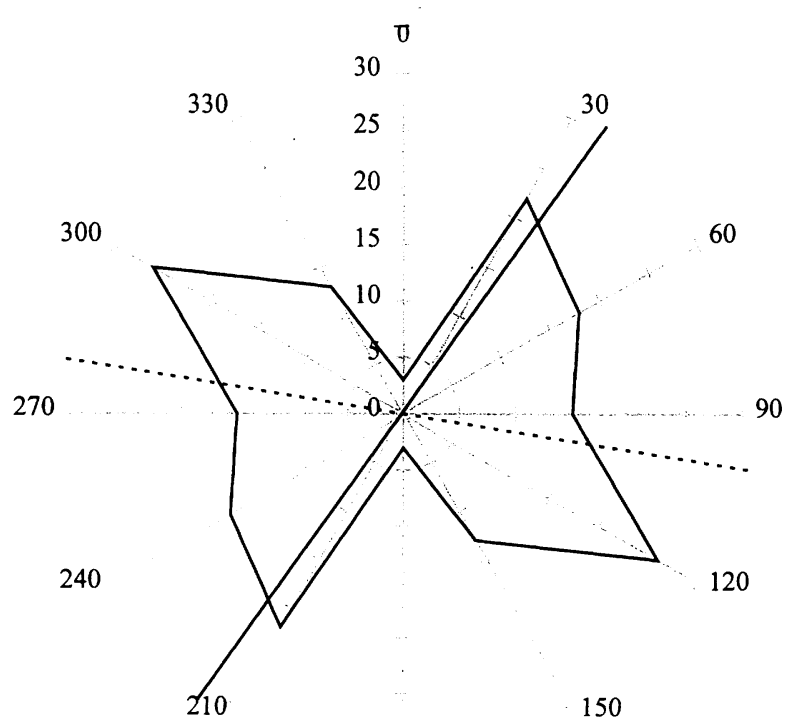

180

Figure 13. Planar orientation distribution histogram for zone $7, Q=4.2 \times 10^{-6} \mathrm{~m}^{3} / \mathrm{s}, \mu=2000 \mathrm{cp}$. - Jeffery's angle; - . - Perpendicular to local flow direction.

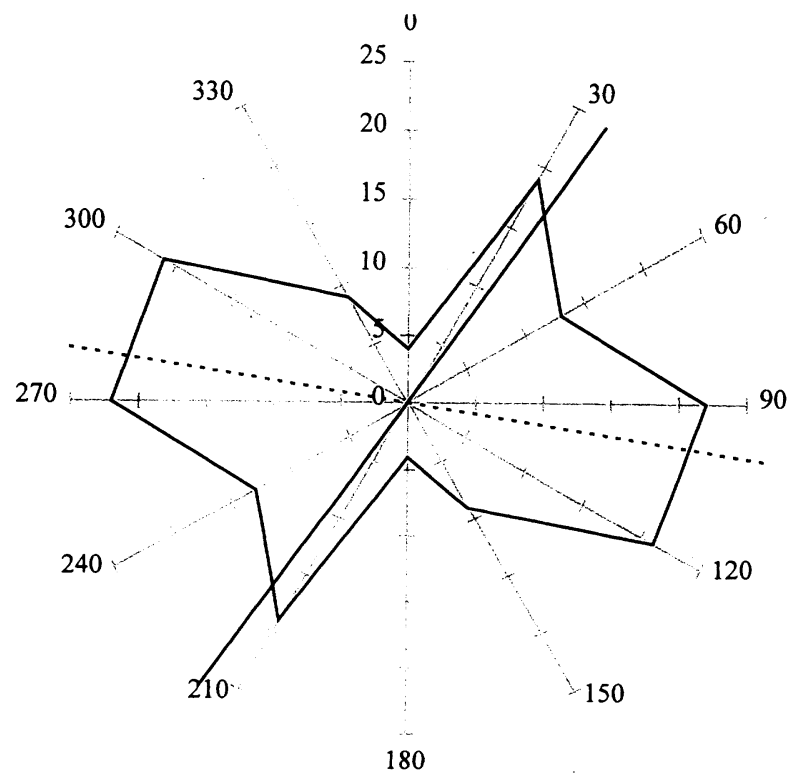

Figure 14. Planar orientation distribution histogram for zone $7, Q=3.3 \times 10^{-6} \mathrm{~m}^{3} / \mathrm{s}, \mu=2000 \mathrm{cp}$. - Jeffery's angle; - . - Perpendicular to local flow direction. 
steady, low Reynold's number, isothermal, Newtonian, and dilute fiber concentration), one would have expected a much better agreement between the experimental and numerical results. In particular, within the zones off the symmetry axis (i.e., zones 1, 2, 6, and 7), both planar and through-thethickness velocity gradients contribute to fiber alignment around the local flow direction. In these zones, bimodal orientation distribution due to the presence of a significant number of fibers aligned perpendicular to the local flow direction is totally unexpected and cannot be explained by the so-called skin-core effect observed through the thickness of numerous injection molded parts. The skin-to-core transition (i.e., the transition from fiber alignment perpendicular to local flow around the midplane to alignment around the local flow direction near the mold walls) is usually due to the planar flow deceleration and is not applicable to zones $1,2,6$, and 7 . The skin-core orientation structure is observed after sudden or gradual expansions of the mold cavity such as those observed around an inlet gate.

The discrepancy between the theoretical predictions and experimental results may be explained by the interactions between the fibers and the mold walls. Those fibers that are oriented around the local flow direction and located near the walls try to tumble infrequently through the thickness due to the higher shear gradients (frequency of the tumbling depends on the fiber aspect ratio and velocity gradients). Based on the fiber size and its location through the thickness, the tumbling may initiate significant mechanical or hydrodynamic interactions with the wall. This interaction will either push the fiber's centroid away from the wall such that the fiber can rotate through the thickness with minimal wall effects or change the orbit of the fiber so that the tumbling becomes more of a planar rocking motion with a smaller through-the-thickness component. This new orbit minimizes wall effects on the fiber's rotation by keeping the fiber orientation nearly perpendicular to the local flow direction. In our experiments, fibers seem to be affected by the walls, which leads to the observed bimodal orientation distribution with an alignment component perpendicular to the local flow.

\section{CONCLUDING REMARKS}

Orientation formation in short-fiber suspensions injected into a planar mold cavity is experimentally studied. The planar fiber orientation field is characterized at nine zones of interest near the sudden contraction. Bimodal orientation distributions are observed in almost all zones under several different flow conditions. Fibers are observed to align either around the direction predicted by Jeffery's theory or around the direction perpendicular to the local flow. Large fiber size combined with wall effects may interfere with Jeffery orbits of the fibers near the top and bottom mold walls and cause the observed alignment perpendicular to the local flow. 


\section{REFERENCES}

Altan, M. C., Subbiah, S., Güçeri, S. I., and Pipes, R. B., 1990, "Numerical Prediction of ThreeDimensional Fiber Orientation in Hele-Shaw Flows," Polymer Eng. Sci., Vol. 30(14), pp. 848-859.

Altan, M. C., and Rao, B. N., 1995, "Closed-Form Solution for the Orientation Field in a Center-Gated Disk,” J. Rheology, Vol. 39(3), pp. 581-599.

Bay, R. S., and Tucker, C. L., 1992a, "Fiber Orientation in Simple Injection Moldings. Part II: Experimental Results," Polymer Composites, Vol. 13, pp. 332-341.

Bay, R. S., and Tucker, C. L., 1992b, "Stereological Measurement and Error Estimates for ThreeDimensional Fiber Orientation,” Polymer Engr. Sci., Vol. 32(4), pp. 240-253.

Bay, R. S., and Tucker, C. L., 1992c, "Fiber Orientation in Simple Injection Moldings. Part I: Theory and Numerical Methods," Polymer Composites, Vol. 13, pp. 317-331.

Chung, S. T., and Kwon, T. H., 1995, "Numerical Simulation of Fiber Orientation in Injection Molding of Short-Fiber-Reinforced Thermoplastics," Polymer Engr. Sci., Vol. 35(7), pp. 604-618.

Chung, S. T., and Kwon, T. H., 1996, "Coupled Analysis of Injection Molding Filling and Fiber Orientation, Including In-Plane Velocity Gradient Effect," Polymer Composites, Vol. 17(6), pp. 859-872.

De Frahan, H. H., Verleye, V., Dupret, F., and Crochet, M. J., 1992, "Numerical Prediction of Fiber Orientation in Injection Molding," Polymer Eng. Sci., Vol. 32(4), pp. 254-266.

Friedrichs, B., Güçeri, S. I., Subbiah, S., and Altan, M. C., 1993, “A Numerical Approach to Short Fiber Reinforced Reaction Injection Molding (RRIM)," J. Mat. Processing \& Manuf. Sci., Vol. 1, pp. 331-349.

He, J., Olivero, K. A., and Altan, M. C., 1997, “Orientation Formation in Planar Mold Filling: Theory and Numerical Predictions,” ASME, Vol. MD-79, Eds. Wang, H.P., Turng, L.-S., and Marchal, J.-M., pp. 309-322.

Jeffery, G. B., 1922, "The Motion of Ellipsoidal Particles Immersed in a Viscous Fluid," Proc. Royal Soc., Vol. A102, pp. 161-179.

Lee, W., and George, H. H., 1978, "Flow Visualization of Fiber Suspensions," Polymer Eng. Sci., Vol. 18(2), pp. 146-156.

Matsuoka, T., Takabatake, J., Inoue, Y., and Takahashi, H., 1990, "Prediction of Fiber Orientation in Injection Molded Parts of Short-Fiber-Reinforced Thermoplastics," Polymer Engr. Sci., Vol. 30(16), pp. 957-966.

Murty, K. N., and Modlen, G. F., 1977, "Experimental Characterization of the Alignment of Short Fibers During Flow,” Polymer Engr. Sci., Vol. 17(12), pp. 848-853.

Olivero, K. A., He, J., and Altan, M. C., 1997, “Orientation Formation in Planar Mold Filling: Experimental Results,” ASME, Vol. MD-79, Eds. Wang, H.P., Turng, L.-S., and Marchal, J.-M., pp. 323-333.

Rao, B. N., 1996, "Rheology and Flow of Fiber Suspensions in Composite Materials Manufacturing," $\mathrm{Ph}$. D. Thesis, University of Oklahoma, Norman, OK.

Vincent, M., and Agassant, J. F., 1985, "Experimental and Theoretical Study of Short Fiber Orientation in Diverging Flows," Rheol. Acta, Vol. 24, pp. 603-610. 